\title{
Anaesthetic Management of Temporomandibular Joint Ankylosis without Fibrotic Bronchoscope- A Review of 31 Cases
}

\author{
*Dr.JyotiKulkarni, \#Dr. Kanchan Shah, \\ Dr. Abdul AhadGhaffarKhan, Dr. Sameer Khaire, \\ *M.D.Anaesthesia ASSISTANTPROFESSOR \\ \#MDS.Oral \&Maxilofacial Surgery...ASSOCIATE PROFESSOR, MDS. Oral \&Maxilofacial Surgery... \\ ASSISTANT PROFESSOR Govt. Dental College Aurangabad.
}

\begin{abstract}
:
Introduction: Temporomandibular joint (TMJ) ankylosisis acondition associated with limited to zero mouth opening. In addition to facial asymmetry, malocclusion, anemia\& malnutrition, airway obstruction may be present. All these changes make not only intubation but ventilation also difficult.

Method: In this article we wantto report anaesthetic management of 31 patients having temporomandibular joint ankylosis by using blind nasal intubation technique. In all patients mouth opening is either zero or less than $5 \mathrm{~mm}$.

Observation: In $87.09 \%$ patients we could intubate by blind nasotracheal intubation technique while $13.91 \%$ patients required tracheostomy.

Conclusion: Blind nasal intubation, fiber optic guided nasal intubation, retrograde intubation \& tracheostomy are the different techniques of securing airway in these patients. With proper preoperative preparation, induction with inhalationalagent \& blind nasal technique for intubation we can manage patients of temporomandibular joint ankylosis successfully. Blind nasal intubation technique can be mastered by practice \& is useful in managing difficultintubation cases particularly when fibrotic bronchoscope is not available
\end{abstract}

Key words: TMJ ankylosis, blind nasal intubation, fiber optic intubation, retrograde intubationtracheostomy.

\section{Introduction}

Temporomandibular joint (TMJ) ankylosis results in inability to open mouth either partial or complete. In India incidence of TMJ ankylosis is still high in. It is observed from 2 years of age to 60 years of age ${ }^{1}$. Congenital TMJ ankylosis is very rare. Trauma or infection is the cause of TMJ ankylosis. Facial asymmetry, malocclusion, anemia, malnutrition may are the consequences. It also leads to increased airway obstruction, obstructive sleep apnea and corpulmonale ${ }^{2}$. Airway obstruction is secondary to structural encroachment on or pharyngeal and hypo pharyngeal lumen, subatmosphericintrapharyngeal pressure and hypotonocity of oropharyngeal muscles ${ }^{3}$. All these structural deformities lead to difficulty in ventilation, intubation \&extubation.

Treatment is always surgical. Condylectomy, gap arthoplasty, interposition arthoplasty\& artificial replacement of joint are the different procedures performed.

Due to nil or limited mouth opening nasotracheal intubation either blind or guided by fiber optic bronchoscope, retrograde intubation or tracheostomy are the safer techniques of securing airway. Awake, Fiber optic scope guided nasotracheal intubation is the safest technique of intubation. As TMJ ankylosisis common in pediatric age group awake intubation is difficult to perform in these patients. Due to unavailability of paediatricfibreoptic bronchoscope we managed all patients by blind nasotracheal intubation. Here we report anaesthetic management of 31 cases of TMJ ankylosis posted for condylectomy and gap arthoplasty under general anaesthesia in last 10 years.

All patients were managed by topical anaesthesia of upper airway followed by light sedation with Inj. Midazolam, Inj. Glycopyrolate\& Inj. Fentanyl. Patients were induced with oxygen, nitrous oxide and halothane. Endotracheal tube was passed through most patent nostril. After confirmation tube was fixed and InjVecuronium was given. Once mouth was opened oral cavity was packed with tape gauge. Depending upon weight and blood loss IV fluids and blood transfusion was given. At the end of surgery reversal was done with Neostigmine and Glycopyrolate. If required patients were observed in post surgical intensive care unit.

In developing countries like India still we do not have modern gadgets like paediatricfibreoptic laryngoscope. The purpose of this article is to stress the importance of blind nasal intubation in management of 
difficult airway particularly in patients having restricted mouth opening in absence of modern gadgets of intubation.

\section{Material \&Method}

Detail preoperative evaluation \& investigations were done. Anaemia was treated with oral iron therapy. In patients having obstructive breathing, nasal obstruction was ruled out by detail local examination., $\mathrm{X}$-ray paranasal sinus, $\mathrm{X}$ - ray neck anteroposterior\& lateral view taken to rule out shift of larynx, trachea. Consent for surgery, anaesthesia, blood transfusion, cricothyroidotomy and tracheostomy was obtained.

After establishment of IV line Lignocaine with adrenaline was instilled in the most patent nostril. Patients were given InjGlycopyrolate $0.05 \mathrm{mg} / \mathrm{kg}$, Inj Midazolam $0.02 \mathrm{mg} / \mathrm{kg}$ and Inj Fentanyl $1 \mu \mathrm{g} / \mathrm{kg}$. Patients were preoxygenated with $100 \% \mathrm{O}_{2}$ for 5 mints. Induction was done with halothane, nitrous oxide \& oxygen. After achieving deep plane of anaesthesia, uncuffednasotracheal tube was passed using breath sounds as guide and then tube was connected to Bains circuit. Another nostril was obliterated with operators fingure and ventilation continued on Bains circuit. Tube was advanced till we could see maximum bag movements. Once patient was completely relaxed small catheter was passed through the tube \& $4 \%$ Lignocain sprayed through small catheter to achieve topical anaesthesia of glottis. Then the tube was advanced till it enters the larynx. Position of tube was confirmed by connecting it to Bains circuit and observation of bag movement. By auscultation air entry was checked and $\mathrm{O}_{2}$ saturation was noted on pulse oxymeter. Once confirmed patient was given Inj. Vecuronium $0.08 \mathrm{mg} . / \mathrm{kg}$ IV. with Nitrous oxide, Oxygen \& halothane. Once mouth was opened the oral cavity was packed with tape gauge to avoid trickling of saliva or blood along with tube.

IV fluids \& blood transfusion were given as per blood loss. At the end of surgery patients were reversed with Inj.Neostigmin (0.06 mg./kg.) and Inj.Glycopyrolate $(0.05 \mathrm{mg} / \mathrm{kg}$.). Decision of extubation was taken depending upon the consciousness of patient and adequacy of respiration.

\section{Observation}

Among all 31patients of TMJ ankylosis $77.41 \%$ were below the age of 16 years and $22.59 \%$ were above the age of 16yrs. Youngest one is of 3 years of age and oldest one is of 45 years. $61.29 \%$ patients were female while $38.71 \%$ patients were male. 51.61\% patient had bilateral TMJ ankylosis and $48.39 \%$ patients had unilateral TMJ ankylosis. In $87.09 \%$ patients we could intubate by blind nasotracheal intubation technique while $13.91 \%$ patients required tracheostomy.

Patients who had facial asymmetry and onset of ankylosis in early childhood were difficult to intubate than adult patients. It might be due to structural deformity of larynx \& trachea, which were shifted to affected side of TMJ ankylosis. $25.81 \%$ patients were observed in post-surgical intensive care unit and extubated within 24 hours of surgery. All these patients were below the age of 10 years. In adult patients we did not observe any difficulty in ventilating patients on mask. In $8 \%$ paediatric patients mask ventilation was difficult after induction of anaesthesia. In these patients nasal tube was passed like nasopharyngeal airway and ventilated till relaxation occurs and then tube was advanced.

TABLE I

AGE DISTRIBUTION :-

\begin{tabular}{|l|l|l|}
\hline AGE & NO. OF PATIENTS & $\%$ OF PATIENTS \\
\hline$<16$ YEARS & 24 & $77.41 \%$ \\
\hline$>16$ YEARS & 7 & $22.59 \%$ \\
\hline
\end{tabular}

TABLE II

SEX DISTRIBUTION :-

\begin{tabular}{|c|c|c|}
\hline SEX & NO. OF PATIENTS & $\%$ OF PATIENTS \\
\hline FEMALE & 19 & $61.29 \%$ \\
\hline MALE & 12 & $38.71 \%$ \\
\hline
\end{tabular}

TABLE III

TYPE OF ANKYOLOSIS:-

\begin{tabular}{|l|c|c|}
\hline TYPE & NO. OF PATIENTS & \% OF PATIENTS \\
\hline UNILATERAL & 16 & $51.61 \%$ \\
\hline BILATERAL & 15 & $48.39 \%$ \\
\hline
\end{tabular}


TABLE IV

METHOD OF INTUBATION :-

\begin{tabular}{|l|l|l|}
\hline METHOD & NO. OF PATIENTS & $\%$ OF PATIENTS \\
\hline BLIND NASAL INTUBATION & 27 & $87.09 \%$ \\
\hline TRACHEOSTOMY & 4 & $13.91 \%$ \\
\hline
\end{tabular}

TABLE V

TIME OF EXTUBATION :-

\begin{tabular}{|l|l|l|}
\hline TIME & NO. OF PATIENTS & $\%$ OF PATIENTS \\
\hline $\begin{array}{l}\text { IMMEDIATE POST } \\
\text { OP. }\end{array}$ & 23 & $74.19 \%$ \\
\hline AFTER 24 HOURS OF SURGERY & 8 & $25.81 \%$ \\
\hline
\end{tabular}
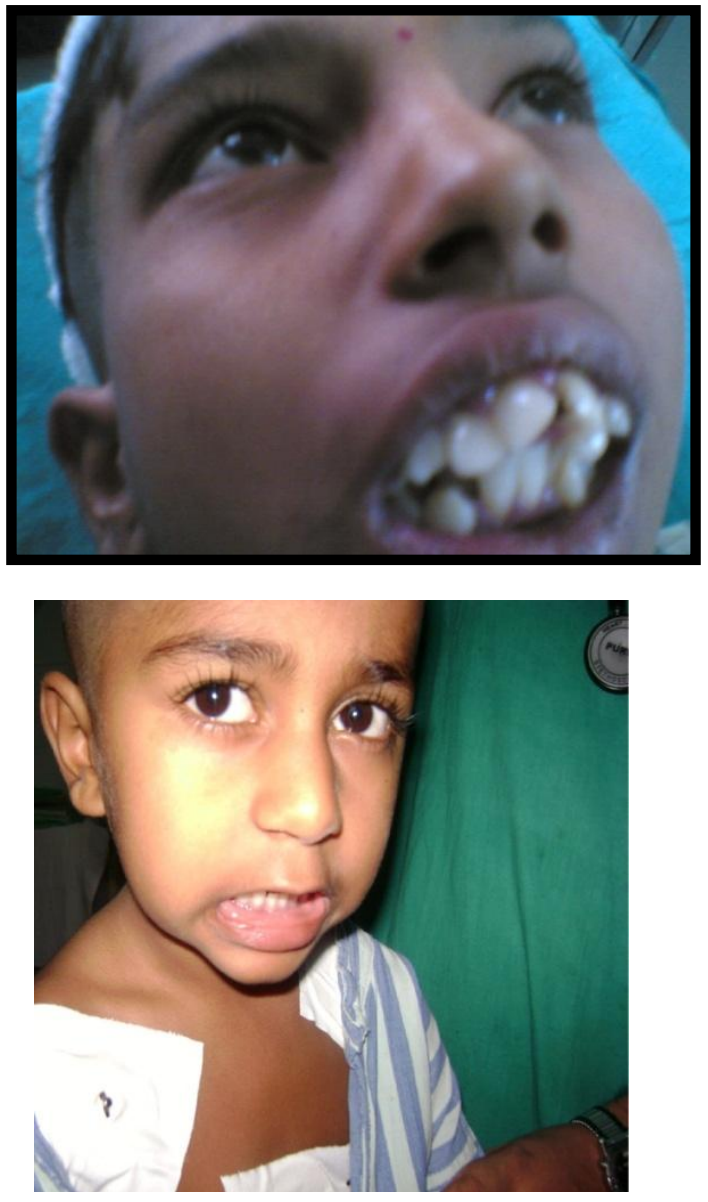

\section{RESTRICTED MOUTH OPENING}

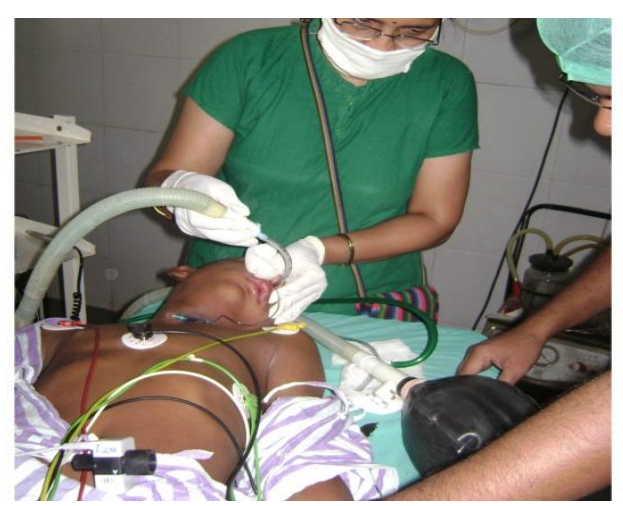




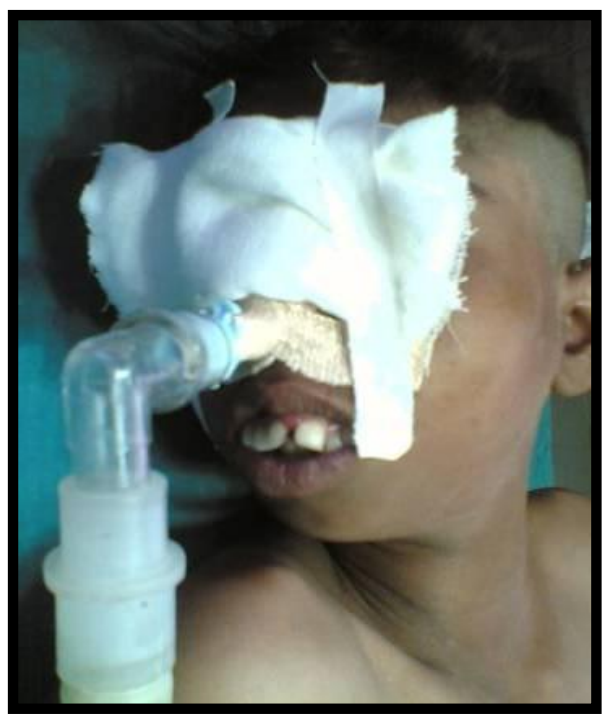

TECHNIQUE OF BLIND NASAL INTUBATION

\section{Discussion}

Temporomandibular joint ankylosis results in restricted or nil mouth opening \& jaw function get affected. It may be unilateral or bilateral. Facial asymmetry, deviation of mandible \& chin on affected side, hypoplastic mandible with receiding chin \& fullness of face on affected side are the features of unilateral TMJ ankylosis ${ }^{1}$. In bilateral TMJ ankylosis facial symmetry is maintained but micrognathia is present. Birdface deformity, receding chin, narrow maxilla, protruding upper incisors with nil or few mms mouth opening are the features of bilateral TMJ ankylosis.

Untreated cases may lead to malnutrition, facial asymmetry, and respiratory distress, and poor oral hygiene, carious or impacted teeth. Increased airway resistance \&corpulmonale may occur ${ }^{2}$. Structural encroachment of oropharyngeal lumen, subatmosphericintrapharyngeal pressure, hypo tonicity of oropharyngeal muscles resulted in airway obstruction. If occurred during growth of child it results in narrow oropharyngeal airway secondary to shortening of mandibular rami and narrowing of space between the mandibular angle.

All these structural abnormalities with restricted or no mouth opening results in difficulty in securing airway. Awake nasal intubation is the safest approach of intubation. Nasal intubation either blind or fibre-optic guided \& awake or under anaesthesia, retrograde intubation \& tracheostomy are the different techniques of securing airway in these patients. If anaesthetic agents are used there is risk of perioperative apnea, desaturation $\&$ dysarhythmia ${ }^{3}$. Due to extreme sensitivity to central depressant drugs benzodiazepines and opioids should be used in titrated dose only ${ }^{3}$.

Retrograde intubation needs patient's cooperation which is difficult to obtain in children. Tracheostomy is indicated in those patients in whom significant postoperative airway compromise is anticipated. Considering severe morbidity, long term side effects \& mortality it should be the last option, only in case of emergency ${ }^{3}$.Use of Gum Elastic boogie to facilitate blind nasal intubation has been suggested ${ }^{4}$. Due to unavailability of fibre-optic bronchoscope we decided to perform blind nasal intubation.

It is difficult to secure airway in children due to small mouth opening. General anaesthesia is necessary before making an attempt to secure airway. Awake intubation needs patients co-operation, local blocks for nerves of larynx and topical anaesthesia for upper airway. There is a chance of severe laryngospasm or inability to pass endotracheal tube. So we used Halothane, Nitrous oxide \& Oxygen along with topical anaesthesia\& light sedation. Topical anaesthesia can be achieved by Cocaine, Lignocain 3\% with $0.25 \%$ Phenylephrine, and Lignocain with adrenaline $e^{5}$. This will provide topical anaesthesia\& vasoconstriction which prevents bleeding \& widen the nasal passage ${ }^{5 \& 6}$. Nebulization with $10 \%$ Lignocaine also provides topical anaesthesia. But it is time consuming, high concentration of Lignocaine is required \& may need supplenmentation ${ }^{6}$. Anticholinergic agents reduce secretions, increase intensity \&speed of onset \& duration of topical anaesthesia ${ }^{7}$. Use of Sevoflurane\&Propofol for induction of anaesthesia is also safe. Induction with Ether \& Halothane has been attempted $^{8}$. It provides muscle relaxation, bronchodilatation\& good intubating condition. Before intubation nasal patency should be tested. If both nostrils are patent Right nostril is chosen as the bevel of tube will face the flat nasal septum \& helps in reducing damage to inferior turbinate ${ }^{9}$.

For blind nasal intubation patient was placed in sniffing position. Lubricated endotracheal tube was introduced through nose in a plane perpendicular to face. Tube was inserted until maximum breath sounds were heard. Tube was connected to Bain's circuit \&Ventilation continued with $\mathrm{N}_{2} \mathrm{O}+\mathrm{O}_{2}$ \& halothane. Another nostril 
was obliterated with operator's finger. Once complete muscle relaxation was achieved a infant feeding tube was passed through nasal tube \& $4 \%$ Lignocaine sprayed through it.

Patient's spontaneous respiration helps to spread the local anaesthetic solution over glottis \& then tube was advanced .

If tube did not enter in glottis manipulation of tube was necessary for correct placement of the endotracheal tube. If tube had entered in esophagus tube was withdrawn \& neck extension was done. If tube was impinged anteriorly slight neck flexion was done. If tube was felt beside larynx turning the tube tip away from that side might help for successful intubation.

Successful intubation was confirmed by auscultation of breath sounds, pulse, $\mathrm{ETCO}_{2} \&$ observation of bag movements. After confirmation injection Vecuronium was given along with $\mathrm{N}_{2} \mathrm{O}+\mathrm{O}_{2}$ \& Halothane. In cases where intubation was failed tracheostomy was performed under halothane \& $\mathrm{N}_{2} \mathrm{O}+\mathrm{O}_{2}$. Nasal intubation is contra-indicating in coagulopathy severe intranasal disorder, basilar skull fracture and C.S.F. leak. Nasal intubation may lead to septicemia, perforation of nasal septum, injury to inferior turbinate.

Transnasalfiberoptic guided intubation under sedation or under the influence of inhalational anaesthetics with patient on spontaneous respiration is the safest approach of intubation ${ }^{10}$. But unavailability of fiber optic bronchoscope is the limiting factor. Retrograde intubation is difficult to perform if mouth opening is less than $5 \mathrm{~mm}$.In children modified technique of retrograde intubation under general anaesthesia has been described $^{10}$.

So blind nasal intubation under effect of halothane, Nitrous oxide and Oxygen with spontaneously breathing patient was the option we had chosen for intubating these patient. Inhalational induction along with topical anaesthesia reduces chances of breath holding, laryngospasm. Hypotension \&bradycardia may occur due to Halothane.

Tracheostomy was the last option only in emergency when all other approaches failed. Tracheostomy has its own advantages \& disadvantages. Surgical airway should be kept reserved for failed intubation .In emergency situation cricothyroidotomy provides effective ventilation.

\section{Conclusion}

We concluded that patients with TMJ ankylosis can be managed without any morbidity \& mortality by blind nasal technique under inhalational \& topical anaesthesia without any modern gadget successfully. In 87.09\% patients we could intubate by blind nasotracheal intubation technique \& $13.91 \%$ patients required tracheostomy. Blind nasal intubation technique should be mastered by all anesthesiologist as it is helpful in managing not only cases of restricted mouth opening but also in other emergency situations of difficult intubation.

\section{References}

[1]. Neelima Anil Malik, Ankylosis of temporomandibular joint \& its management. Ch. No. 22, Text Book of oral \& maxillofacial surgeryFirst Edition, 207-219, 2002

[2]. SOAJike, MDChom,VIAMaoyeiw, etall,Nonsyndromal,true congenital ankylosis of temporomandibular joint-A case report. West Indian Med J 2006, 55-6-444

[3]. Shah F R,Sharma R K, Hillowall R N ,Karandikar A D, J Indian Soc. PedoPrev Dentistry 2002 ;20;1,16-20

[4]. AroraMk ,Karamchandani K, Trikha A, Use of Gum Elastic bougie to facilitate blind nasotracheal intubation in children - A series of 3 cases -A case report. Anaesthesia, March 2006- 61- 3; 291- 294

[5]. Thomas J Gal Airway management ,ch. No. 42, Millers Anaesthesia by Ronald Miller, Sixth edition 2005, vol 2, 1617 - 1652

[6]. Nick Woodall, Fibre-optic intubation including local anaesthesia for awake intubation, Anaesthesia\& intensive care medicine, vol 6:8 Aug 2005, 22-26

[7]. NJH Davies, J N Cashmab, Airway management ch. No. 2.6 Lees synopsis of Anaesthesia, 201 -2302006, 13 ${ }^{\text {th }}$ edition

[8]. Vas L, Sawant P A review of anaesthetic technique in 15 paediatric patients with temporomandibular joint ankylosis. Paediatricanaesthesia 2001,March 11:2:237-244

[9]. DrYatrindrakumarBatra ,DrPreethi J Maytew , Airway management with endotracheal intubation including awake intubation \& blind intubation. Indian J of AnaesthesiaVol 49-No 4 ; Aug 2005 -263-268

[10]. ShailaKamat, MithunRaju,Ruchita Gupta, SatishKamat, Modified technique of retrograde intubation in TMJ ankylosis ,Indian J of AnaesthesiaVol 52-2, 196-198 